Proceedings

\title{
The Nutraceutical Properties of "Pizza Marinara TSG" a Traditional Food Rich in Bioaccessible Antioxidants ${ }^{+}$
}

\author{
Irene Dini *, Luana Izzo, Giulia Graziani and Alberto Ritieni \\ 1 Department of Pharmacy, University of Naples Federico II, Via Domenico Montesano 49, 80141 Napoli, \\ Italy; luana.izzo@unina.it (L.I.); giulia.graziani@unina.it (G.G.); alberto.ritieni@unina.it (A.R.) \\ * Correspondence: irdini@unina.it \\ + Presented at the 1st International e-Conference on Antioxidants in Health and Disease, 01-15 December \\ 2020; Available online: https://cahd2020.sciforum.net/.
}

Published: 30 November 2020

\begin{abstract}
Italian gastronomy experiences have ever-enhancing fame around the world. It is due to the linkage between taste and salubriousness commonly related to Mediterranean foods. The market proposes many types of pizza to suit all palates. In this work, the antioxidant potential of the "pizza "marinara" included in the register of traditional specialties guaranteed (TSG) was determined. ABTS method evaluated the antioxidant activity of the pizza homogenized. In vitro digestion models estimated the intestinal and gastric bioaccessibility of the main antioxidant compounds (lycopene and phenolics). To our knowledge, this is the first study to provide the content, antioxidant potential, and bioaccessibility of the antioxidants (polyphenols and lycopene) contained in the traditional pizza "marinara TSG". Our results showed that the "pizza marinara TSG" had polyphenols concentration, lycopene level, antioxidant activity, and bioaccessibility of phenolic compounds and lycopene better than other similar pizzas. They confirmed the nutritional importance of traditional preparations and established the functional potential of "pizza marinara TSG" as a food rich in bio-accessible antioxidants.
\end{abstract}

Keywords: Pizza Napoletana; lycopene; polyphenols; bioaccessibility; nutraceutical properties; antioxidant activity

\section{Introduction}

Italian gastronomy experiences have ever-enhancing fame around the world. It is due to the linkage between taste and salubriousness commonly related to Mediterranean foods. Pizza is a universal, appreciated product of the Italian gastronomy. It was cooked in southern Italy for the first time (before $1000 \mathrm{AD}$ ). The pizza was created for poor people with inexpensive ingredients flour, yeast, water, edible oil, and salt. In the 16th century, when the tomato was imported from America, pizza became as we know it today [1]. In 1750, was cooked the first "marinara" (tomato, garlic, oregano, oil) and in 1850, the first "Margherita" (tomato, mozzarella, oil) [1]. In 1889, the pizza maker Raffaele Esposito added the basil on the "pizza Margherita", giving it the colors of the Italian flag [1]. Recently the food market has grown, offering novel chances to catering. Pizza has become an international food. Several imitations have been made. New kind of pizzas demands their own identities. They are daughters of the times and denote the commercial value of the product. However, they often have nothing to do with the Neapolitan pizza, the result of the traditional Mediterranean 
The 1st International Electronic Conference on Antioxidants in Health and Disease, 1-15 December 2020

culinary knowledge. These pizzas differ from the original product in both organoleptic and nutritional characteristics. Some products are of poor quality and damage the reputation of the original product. Traditional foods represent the identity, culture, history, local economy, heritage and are essential elements for the dietary patterns of a country. Pizza has a crucial role in the traditional habits of Italian culture. Several certificates and labels have been assigned to preserve the Neapolitan pizza identity. The name "pizza Napoletana" has been entered in the register of Traditional Specialties Guaranteed (TSG) [2]. TSG is a tolerant European Union (EU) food designation. It designates foods made with "traditional" techniques or ingredients with a proven usage on the Community market for at least 25 years. TSG makes the product distinguishable from other similar products, and the consumers are informed based on its characteristics. In 2015 the Italian Ministry of Agricultural, Food, and Forestry Policies inserted the Neapolitan pizza in the list of traditional agri-food products [3]. The "Pizza Napoletana" is an elastic, soft, and easily foldable food with a distinctive savory. It is due to the typical taste of well-cooked bread, tomato, baked mozzarella, and flavor (garlic, oregano, basil). The name "Pizza Napoletana" is limited to "marinara" (tomato, oregano, extra virgin olive oil, and garlic) and "margherita" (tomato, grated cheese, mozzarella or fior di latte, extra virgin olive oil, and basil) pizzas made according to precise guidelines and with traditional ingredients. Flour, yeast, water, edible oil, and salt are kneaded. The dough ferments twice. The first fermentation lasts $2 \mathrm{~h}$; then, the dough is portioned into balls that ferment for another 4-6 h at room temperature. Successively the balls are stretched with the hands, guaranteeing to leave a denser edge on the outer part. Finally, the toppings are added, and the pizza is baked in a wood oven at $485{ }^{\circ} \mathrm{C}$ for $60-90 \mathrm{~s}$. The cooking methods of these foods have been handed down from generation. Lifestyle changes are affecting eating habits, and new pizza is changing from the traditional product. Some traditional preparations have shown health properties that have been tested over time [4,5]. An indicator of the potential benefits of food preparations is the antioxidant activity of compounds in food and their possible synergistic interactions. The antioxidant potential of food is linked to the combined action of phenolic compounds carotenoids, and vitamins (C and E). Natural antioxidants have anti-inflammatory, antioxidant, anti-allergic, anti-atherogenic, antithrombotic, cardioprotective, and antimicrobial effects [6,7]. Nutraceutical values are useful parameters to promote culinary products. Nutraceutical data of composite foods are essential to prepare the basis of dietary recommendations. Regarding pizza, there are no data about its nutraceutical potential. The antioxidant data of composite foods are often calculated from the corresponding individual ingredients, do not consider the transformations produced by cooking techniques, and above all, what happens in the human organism and the real bioaccessibility of these molecules. It is essential to investigate the possible interaction of these compounds and the bioaccessibility within our body to get an idea of their possible beneficial effects. The antioxidant potential of the "Pizza Napoletana" principally depends on the bioavailability of the phenolic compounds and lycopene contained in EVOO and tomatoes. The pizzerias that do not follow the "Pizza Napoletana" production specification replace extra virgin olive oil (EVOO) with other less expensive such as other vegetable oils or olive oil, a mixture of refined olive oil and virgin olive oil. The type of oil used, influences, not only the sensorial [8,9], and technological properties [10], but above all, affects the nutritional and functional properties of the pizza [11]. The oil composition differs in the profile and content of unsaturated fatty acids and the presence of antioxidant molecules. During cooking, unsaturated fatty acids oxidase differently. Compared to other oil, EVOO shows higher resistance to lipid oxidation for the presence of polyphenols [12-16]. Thermal oxidation of heated oils produces free radicals associated with the pathogenesis of many diseases, including cardiovascular diseases and atherosclerosis [17-24]. Tomato is another essential ingredient of the pizza. The tomato sauce is used to prepare traditional pizza TSG. New pizzas are made both with fresh cherry tomatoes and tomato sauce. The treatment of the tomatoes to produce tomatoes sauce modify the physicochemical attributes (color, viscosity, total soluble solids) [22], the pH, and some product quality parameters such as lycopene content in the tomato sauce [25-27]. Lycopene is a carotenoid with antioxidant properties able to decrease the risk of hypercholesterolemia, 
The 1st International Electronic Conference on Antioxidants in Health and Disease, 1-15 December 2020 atherosclerosis, cancer, osteoporosis, infertility, metabolic syndrome, and liver damage [28,29]. In this work, the lycopene level, the antioxidant activity, and the bioaccessibility of lycopene and polyphenols in pizza "marinara TSG" and other similar pizzas not subjected to the production disciplinary were detected to highlight the functional properties of the pizza "marinara TSG".

\section{Experiments}

The pizza "marinara TSG" was prepared according to the production disciplinary of the authentic Neapolitan Pizza Association called “Associazione Verace Pizza Napoletana” or A.V.P.N. The other pizzas like pizza "marinara TSG" varied for oil type (soybean oil, sunflower oil, and olive oil) and tomato sauce or fresh tomato (cherry tomato) used in the recipe. The pizzas were cooked in a wood oven, weighed, homogenized (Ultra Turrax T25 homogenizer, IKA-Werke, Wilmington, NC, U.S.A.), and stored at $-18^{\circ} \mathrm{C}$ until the analysis.

\subsection{Chemicals}

Chemicals and enzymes were bought from Sigma Aldrich (St. Louis, MO, USA) unless specified differently.

Artificial saliva was obtained by mixing $\mathrm{KCl}(89.6 \mathrm{~g} / \mathrm{L}), \mathrm{KSCN}(20 \mathrm{~g} / \mathrm{L}), \mathrm{NaH}_{2} \mathrm{PO}_{4}(88.8 \mathrm{~g} / \mathrm{L})$, $\mathrm{NaSO}_{4}(57 \mathrm{~g} / \mathrm{L}), \mathrm{NaCl}(175.3 \mathrm{~g} / \mathrm{L}), \mathrm{NaHCO}_{3}(84.7 \mathrm{~g} / \mathrm{L})$, urea $(25 \mathrm{~g} / \mathrm{L})$, and $\alpha$-amylase $(290 \mathrm{mg}) \mathrm{in} 80 \mathrm{~mL}$ purified water. The $\mathrm{pH}$ of the solution was adjusted to 2 with $\mathrm{HCl} 6 \mathrm{~N}$ (Raiola et al., 2012).

\subsection{Lycopene Extraction and Quantification}

$6 \mathrm{~g}$ of the homogenized sample was extracted with $100 \mathrm{~mL}$ of hexane in an orbital shaker (Infors AG CH-4103, Bottmingen, Switzerland) for $2 \mathrm{~min}$ at 21,500 rpm. Extraction was repeated until the residue was devoid of color. The extracts were centrifuged at $4000 \times \mathrm{g}$ rpm for $5 \mathrm{~min}$. in a centrifuge professional mod. N.E.Y.A. 10 (Neya Centrifuges Carpi (MO), and the supernatants were filtered with RC $0.45 \mu \mathrm{m}$ microfilters (Whatman ${ }^{\circledR}$ regenerated cellulose membrane filters, Global Life Sciences Solutions, Marlborough, MA, USA). Lycopene was quantified spectrophotometrically $(\lambda 502$ and $\lambda$ $472 \mathrm{~nm}$ ) in a spectrophotometer (Lambda 25, PerkinElmer, Italy) [30].

\subsection{Total Phenolics Extraction and Quantification}

$3 \mathrm{~g}$ of homogenized pizza was extracted with $30 \mathrm{~mL}$ of methanol/water $(70: 30, v / v)$. The extraction procedure was repeated twice for each sample. The mixtures were centrifuged at $4000 \times g$ rpm in a centrifuge professional mod. N.E.Y.A. 10 (Neya Centrifuges Carpi (MO) - Italy), filtered through a Whatman filter paper (Whatman ${ }^{\circledR}$ filters, Global Life Sciences Solutions, Marlborough, MA, USA) and then used for antioxidant activity assay.

Total polyphenol content was measured using the Folin-Ciocalteu colorimetric method described previously by Gao et al. (2000) [30]. Polyphenolic extracts $(0.1 \mathrm{~mL})$ were mixed with FolinCiocalteu reagent $(0.2 \mathrm{~mL})$ and $\mathrm{H}_{2} \mathrm{O}(2 \mathrm{~mL})$ and incubated at room temperature for $3 \mathrm{~min}$. Successively, $20 \%$ sodium carbonate $(1 \mathrm{~mL})$ was added to the mixture, and after $1 \mathrm{~h}$ of incubation at room temperature, the total polyphenols were determined spectrophotometrically $(\lambda 765 \mathrm{~nm})$ in a spectrophotometer (Lambda 25, PerkinElmer, Italy). The results were expressed as gallic acid equivalents (G.A.E.), milligrams per $100 \mathrm{~g}$ of sample. All determinations were performed in triplicate $(n=3)$.

\subsection{Antioxidant Activity Assay.}

The antioxidant assay was performed by the ABTS method, as described by Re et al. (1999) [31]. $7 \mathrm{mM}$ ABTS and $2.45 \mathrm{mM}$ potassium persulfate were left at room temperature $\left(23^{\circ} \mathrm{C}\right)$ in the dark for $16 \mathrm{~h}$. The filtered sample was diluted with $70 \%$ methanol giving $20-80 \%$ inhibition of the blank absorbance with $0.1 \mathrm{~mL}$ sample. $1 \mathrm{~mL}$ of ABTS solution (absorbance $=0.700 \pm 0.050$ ) was added to 0.1 
The 1st International Electronic Conference on Antioxidants in Health and Disease, 1-15 December 2020

$\mathrm{mL}$ of the tested samples and mixed thoroughly. The reaction mixture was left at room temperature for $2.5 \mathrm{~min}$, and successively the absorbance was measured at $\lambda 734 \mathrm{~nm}$ in a spectrophotometer (Lambda 25, PerkinElmer, Italy). Trolox standard solution (final concentration 0-15 M) in methanol was assayed at the same conditions. Results were expressed as Trolox equivalent antioxidant capacity (T.E.A.C., mmol Trolox equivalents) on $100 \mathrm{~g}$ of sample.

\subsection{Digestion Procedure.}

All samples were subjected to the in vitro digestion model, as reported by Raiola et al. (2012) [32]with slight modifications. Each sample was mixed with saliva/pepsin/ $\mathrm{HCl}$ digestion for $2 \mathrm{~h}$ at 37 ${ }^{\circ} \mathrm{C}$

\subsection{Duodenal Digestion Simulation}

The samples were mixed with $6 \mathrm{~mL}$ of artificial saliva (immediately after its preparation), $0.5 \mathrm{~g}$ of pepsin $(14,800 \mathrm{U})$ and $\mathrm{HCl} 0.1 \mathrm{~N}$, incubated for $2 \mathrm{~h}$ at $37^{\circ} \mathrm{C}$, and blended in an orbital shaker (Infors AG CH-4103, Bottmingen, Switzerland) at $55 \mathrm{rpm}$.

\subsection{Pancreatic Digestion Simulation}

The sample $\mathrm{pH}$ was brought to 6.5 with $\mathrm{NaHCO}_{3} 1 \mathrm{~N}$ and successively added with $5 \mathrm{~mL}$ (1:1; $v / v)$ of pancreatin $(8 \mathrm{mg} / \mathrm{mL})$, bile salts $(50 \mathrm{mg} / \mathrm{mL})$ and water $(20 \mathrm{~mL})$. The solution was incubated at $37^{\circ} \mathrm{C}$ for $2 \mathrm{~h}$ and blended in an orbital shaker (Infors AG CH-4103, Bottmingen, Switzerland) at 55 rpm. $30 \mathrm{~mL}$ of the mixture was centrifuged at $4000 \times \mathrm{g} \mathrm{rpm}$ at $4{ }^{\circ} \mathrm{C}$ for $1 \mathrm{~h}$ in a centrifuge professional mod. N.E.Y.A. 10, (Neya Centrifuges Carpi (MO)-Italy). The supernatant (bio-accessible fraction) was collected, and the concentration of the lycopene and the total phenolics were evaluated according to the methods described previously.

\subsection{Statistical Analysis}

Significant differences between mean values were determined by performing a one-way ANOVA test (significant level $p<0.05$ ) (@Risk 5.5.1 software package. Palisade, Australia).

\section{Results}

The content, antioxidant activity, and bioaccessibility of lycopene and polyphenols were evaluated in pizzas made with various kind of oil, and different tomato quality. The first step was the evaluation of the concentration of phenols in pizzas "marinara" made with similar procedures (fermentation of dough and cooking modality), but with different oil (soybean, sunflower, olive, and EVOO) and tomato quality (sauce and cherry tomatoes). Then the results were compared with those of pizzas prepared without oil (Control). In general, the addition of oil increased the content of phenolic compounds in all the pizza "marinara" tested, except for the pizza prepared with sunflower and cherry oil (Figure 1). La pizza "marinara TSG" showed a content of phenolic compounds higher than that of the other pizzas. 
The 1st International Electronic Conference on Antioxidants in Health and Disease, 1-15 December 2020

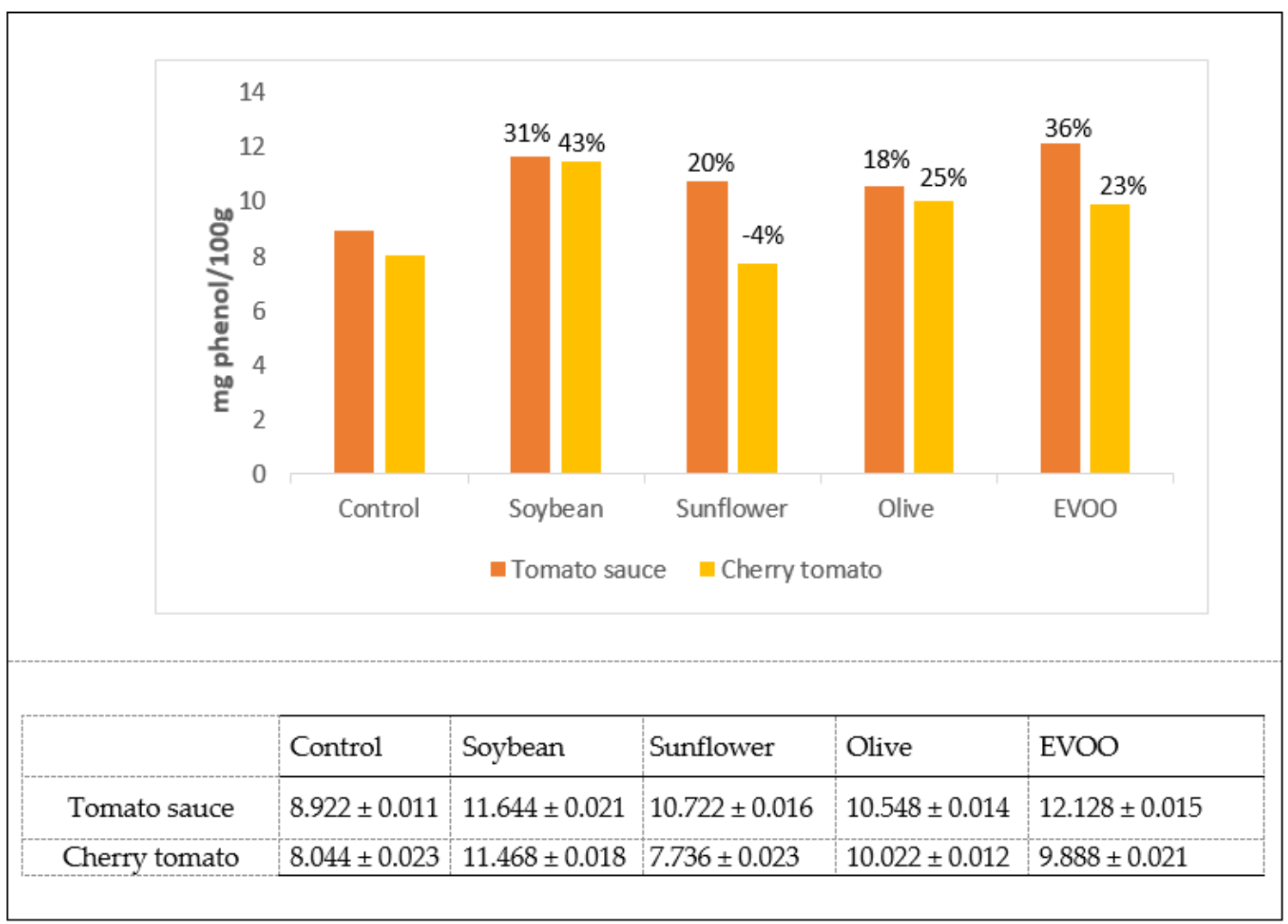

All the measures are statistically significant $(p<0.05)$.

Figure 1. Amount of phenols expressed as $\mathrm{mg} / 100 \mathrm{~g}$ of pizza measured on lipophilic extracts as a function of the type of oil and tomato used.

Successively was determined the antioxidant potential of the different pizzas "marinara". The addition of oil increased the antioxidant activity of the pizzas. The "marinara TSG" had the highest potential (Figure 2). 


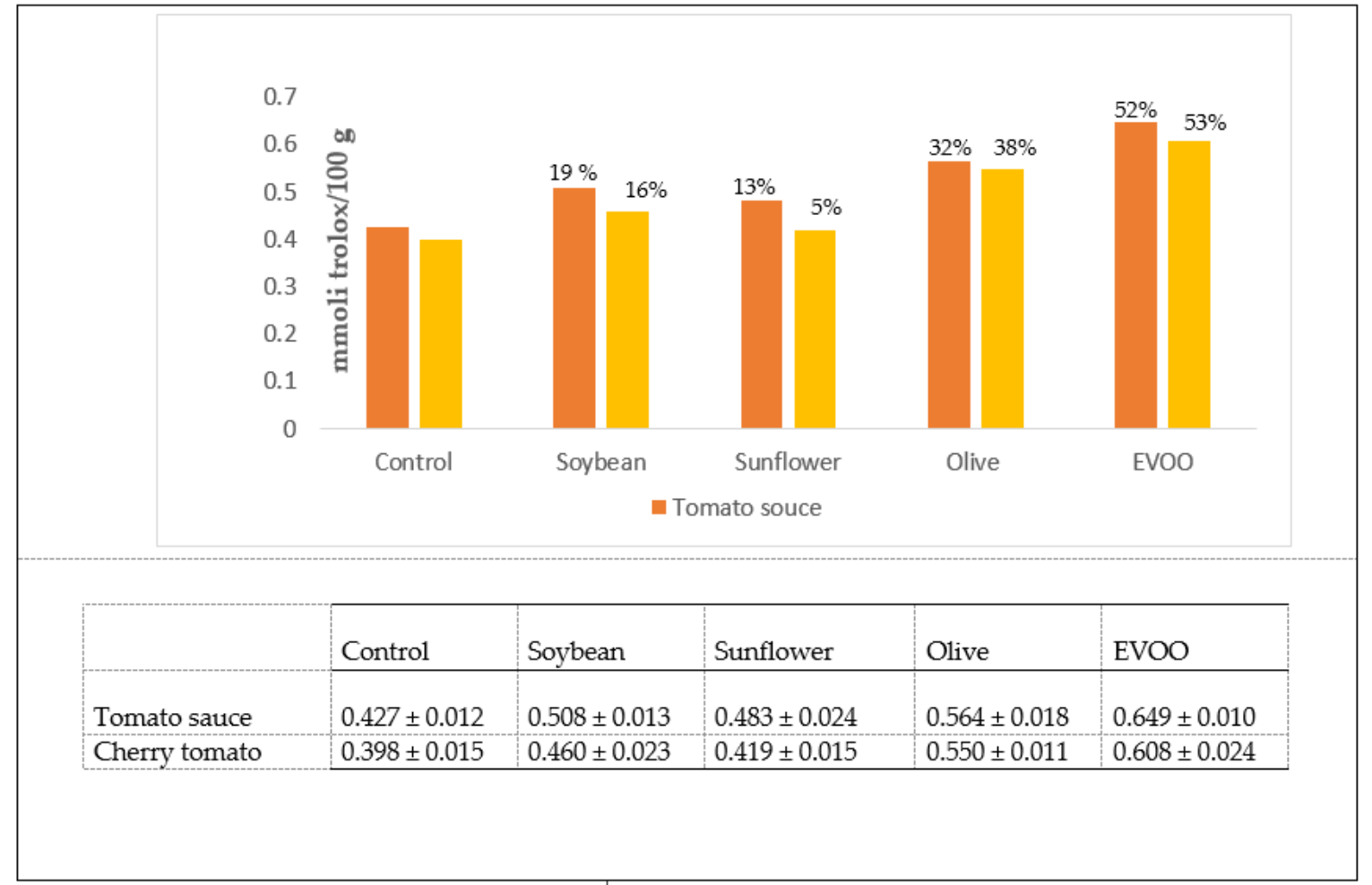

All the measures are statistically significant $(p<0.05)$.

Figure 2. Antioxidant activity of the polyphenolic extracts.

Finally, the bioaccessibility of the phenolic compounds at gastric and intestinal levels was tested (Table 1). The gastric digestion increases the bioaccessibility of total polyphenols in pizzas made with oil. The transition from the gastric (acid environment) to the intestinal (mild alkaline environment) caused much more increase in the bioaccessibility of total polyphenols, especially those contained in pizza "marinara TSG".

Table 1. Bioaccessibility of polyphenols in the pizzas "marinara".

\begin{tabular}{ccccc}
\hline Sample & $\begin{array}{c}\text { mg } \\
\text { Polyphenols/5 } \\
\text { gr of Pizza }\end{array}$ & $\begin{array}{c}\text { \% Polyphenols } \\
\text { Released (Gastric } \\
\text { Phase) }\end{array}$ & $\begin{array}{c}\text { \% Polyphenols Released } \\
\text { (Intestinal Phase) }\end{array}$ & $\begin{array}{c}\text { \% Polyphenols } \\
\text { Residual Pellet }\end{array}$ \\
\hline ControlT & $0.4461 \pm 0.021$ & 5 & 46 & 48 \\
ControlC & $0.4022 \pm 0.019$ & 5 & 41 & 54 \\
SoybeanT & $0.5823 \pm 0.034$ & 10 & 69 & 21 \\
SoybeanC & $0.5734 \pm 0.021$ & 15 & 60 & 25 \\
SunflowerT & $0.5361 \pm 0.012$ & 12 & 60 & 28 \\
SunflowerC & $0.3868 \pm 0.018$ & 13 & 58 & 29 \\
OliveT & $0.5274 \pm 0.017$ & 9 & 71 & 20 \\
OliveC & $0.5010 \pm 0.012$ & 15 & 69 & 16 \\
EVOOT & $0.6064 \pm 0.078$ & 13 & 68 & 14 \\
EVOOC & $0.4944 \pm 0.075$ & 18 & 68 & 14 \\
\hline
\end{tabular}

All the measures are statistically significant $(p<0.05)$. ControlT: Pizza made with tomato sauce, oregano, and garlic; ControlC: Pizza made with cherry tomato, oregano, and garlic;.SoybeanT: Pizza made with tomato sauce, oregano, garlic, and soybean oil: SoybeanC: Pizza made with cherry tomato, oregano, garlic, and soybean oil; SunflowerT: Pizza made with tomato sauce, oregano, garlic, and sunflower oil; SunflowerC: Pizza made with cherry tomato, oregano, garlic, and sunflower oil; OliveT: Pizza made with tomato sauce, oregano, garlic, and olive oil; OliveC: Pizza made with cherry tomato, oregano, garlic, and olive oil;EVOOT (pizza "marinara TSG"): Pizza made with tomato sauce, oregano, garlic, and EVOO; EVOOC. Pizza made with cherry tomato, oregano, garlic, and EVOO. 
The 1st International Electronic Conference on Antioxidants in Health and Disease, 1-15 December 2020

Similarly, the content, antioxidant activity, and bioaccessibility of lycopene were studied. In the same way, to what was happen for the polyphenol content, the lycopene increased in the pizzas prepared with the addition of oil, and the pizza "marinara TSG" showed the highest lycopene content (Figure 3).

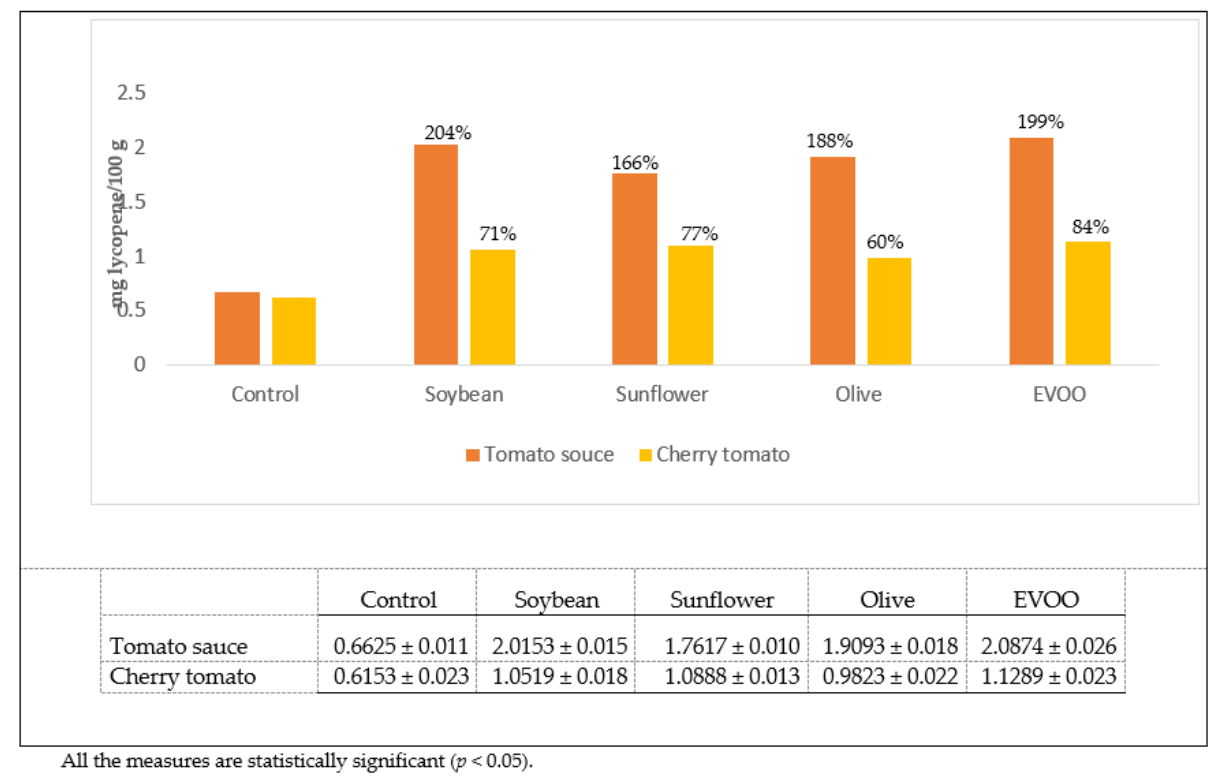

Figure 3. Amount of lycopene expressed as mg/100 $\mathrm{g}$ of pizza measured on lipophilic extracts as a function of the type of oil used.

The antioxidant activity of pizzas prepared with soy and olive oil decreased compared to pizzaprepared without oil. Pizza "marinara TSG" had the highest antioxidant potential (Figure 4).

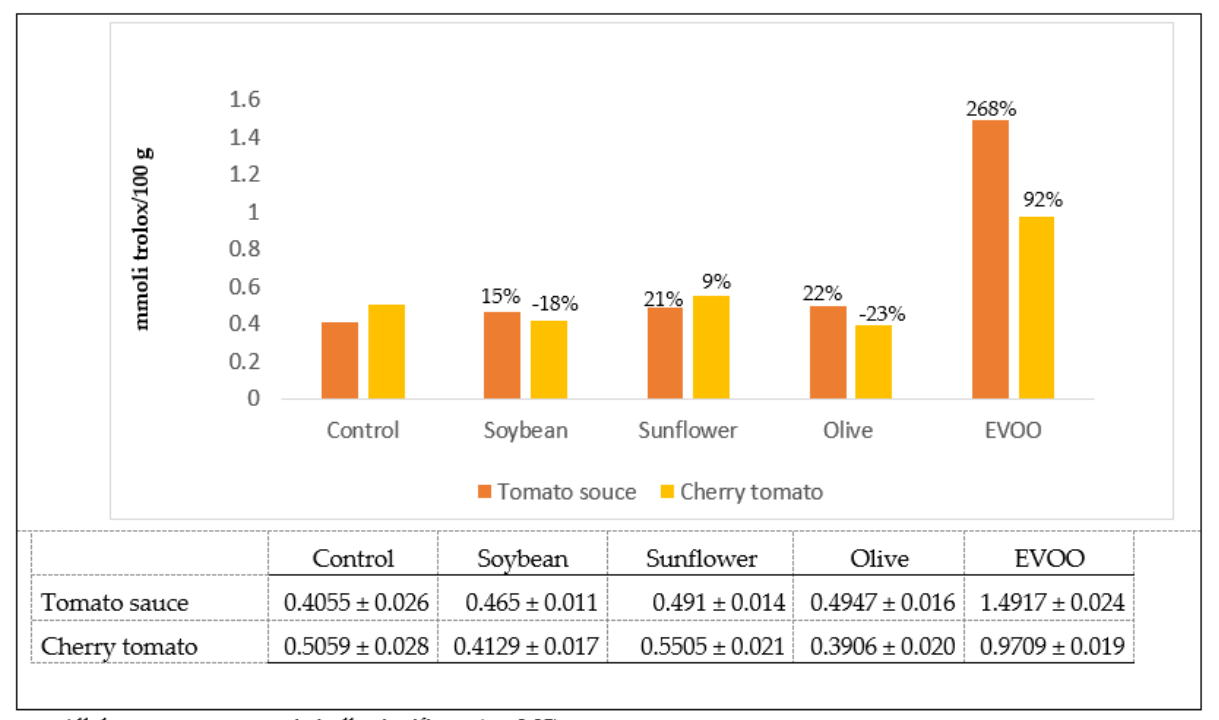

All the measures are statistically significant $(p<0.05)$.

Figure 4. Antioxidant activity of the carotenoid extracts.

The bioaccessibility of lycopene was reported in Table 2. Lycopene was released both gastric ally and intestinally. As already happened for polyphenols, the acid $\mathrm{pH}$ favored the release more. Unlike polyphenols, however, higher concentration of lycopene remained undigested. The lycopene contained in the pizza "marinara TSG" was the most bioaccessible. 
The 1st International Electronic Conference on Antioxidants in Health and Disease, 1-15 December 2020

Table 2. Bioaccessibility of lycopene in the pizzas "marinara" expressed as percentage released at the gastric and intestinal level.

\begin{tabular}{ccccc}
\hline Sample & $\begin{array}{c}\text { mg Lycopene/5 gr } \\
\text { of Pizza }\end{array}$ & $\begin{array}{c}\text { \% Lycopene Released } \\
\text { (Gastric Phase) }\end{array}$ & $\begin{array}{c}\text { \% Lycopene Released } \\
\text { (Intestinal Phase) }\end{array}$ & $\begin{array}{c}\text { \% Lycopene } \\
\text { Residual Pellet }\end{array}$ \\
\hline ControlT & $0.03312 \pm 0.002$ & 9 & 16 & 75 \\
ControlC & $0.03076 \pm 0.001$ & 11 & 12 & 77 \\
SoybeanT & $0.08482 \pm 0.003$ & 15 & 26 & 59 \\
SoybeanC & $0.05259 \pm 0.002$ & 15 & 23 & 62 \\
SunflowerT & $0.08808 \pm 0.001$ & 13 & 26 & 61 \\
SunflowerC & $0.05443 \pm 0.002$ & 20 & 23 & 57 \\
OliveT & $0.09546 \pm 0.004$ & 22 & 33 & 45 \\
OliveC & $0.04911 \pm 0.003$ & 29 & 31 & 40 \\
EVOOT & $0.09907 \pm 0.003$ & 21 & 35 & 44 \\
EVOOC & $0.05644 \pm 0.005$ & 28 & 33 & 39 \\
\hline
\end{tabular}

All the measures are statistically significant $(p<0.05)$. ControlT: Pizza made with tomato sauce, oregano, and garlic; ControlC: Pizza made with cherry tomato, oregano, and garlic; SoybeanP: Pizza made with tomato sauce, oregano, garlic, and soybean oil: SoybeanC: Pizza made with cherry tomato, oregano, garlic, and soybean oil; SunflowerT: Pizza made with tomato sauce, oregano, garlic, and sunflower oil; SunflowerC: Pizza made with cherry tomato, oregano, garlic, and sunflower oil; OliveT: Pizza made with tomato sauce, oregano, garlic, and olive oil; OliveC: Pizza made with cherry tomato, oregano, garlic, and olive oil; EVOOT ("pizza marinara" TSG): Pizza made with tomato sauce, oregano, garlic, and EVOO; EVOOC. Pizza made with cherry tomato, oregano, garlic, and EVOO.

\section{Discussion}

In recent years, there has been a new interest in traditional cuisine and traditional foods rich in antioxidant compounds useful for preventing some chronic-degenerative diseases that cause death in our society, such as cardiovascular diseases and cancer. Crucial nutritional information to define the daily intake of the population and their association with the effects on health is determined by the content of antioxidant compounds and their bioavailability. This study compares the concentrations of some parameters of nutraceutical interest present in the pizza " marinara TSG", and other pizzas called pizza "marinara" but not prepared according to the production disciplinary of TSG. Making Neapolitan pizza TSG is an art that respects the use of indicated ingredients, techniques, and methodologies strictly regulated. The preparation of the pizza varies according to the typology of oil and tomato used. These two products influence the nutrient profile of the pizzas as they bring different concentrations of polyunsaturated fatty acids, phenolics, carotenoids, and other compounds useful for human health. The "pizza Napoletana" is made exclusively in woodovens at $485^{\circ} \mathrm{C}$ for $60-90 \mathrm{~s}$. The temperature reached by pizza is $\sim 204-288^{\circ} \mathrm{C}$. The thermal process produces lipid oxidation, caramelization, and Maillard reaction. The unsaturated fatty acids contained in the vegetable oils oxidize and change their compositions and nutritional value. The oxidation of fatty acids depends on fatty acid composition, polyphenols profile and content [33]. In this work, the addition of oil on the pizza positively contributed to the total polyphenol content. The polyphenolic content and antioxidant activity of pizza "marinara TSG" was superior to other pizzas. The phenolic composition of the oils used to make pizzas was very variable. In the EVOO, there are five different classes of phenolic compounds (secoiridoid, phenolic alcohols, phenolic acids, and flavonoids) [34]. Among these, the secoiridoid were the most abundant [35]. During the cooking, the secoiridoids hydrolyzed. Nevertheless, the antioxidant potential of pizza remains high as their hydrolysis products (tyrosol and hydroxytyrosol) have antioxidant activity [36]. In vitro tests determined the phenolic concentrations that are absorbed and available for physiological functions. The bioaccessibility is an essential prerequisite for the bioavailability. It indicates the level of bioactive compounds that are solubilized in chyme (supernatant) after each step of digestion and that are potentially available for absorption. In vitro digestion simulates the human gastric and intestinal digestion. In vitro tests are faster, less expensive, and offer better controls of experimental variables 
The 1st International Electronic Conference on Antioxidants in Health and Disease, 1-15 December 2020

than in vivo studies [37]. The simulation of the gastric digestion was obtained, adding pepsin and acidifying the samples to $\mathrm{pH} 2$ (the gastric $\mathrm{pH}$ of an adult). The acidification of the samples prevents the denaturation of pepsin that occurs at $\mathrm{pH} \geq 5$. The intestinal digestion was mimicked by neutralizing the sample ( $\mathrm{pH}$ 5.5-6), adding a pancreatin and bile salts (emulsifiers), and finally readjusted to $\mathrm{pH}$ 6.5. The polyphenols contained in the pizzas were released only in small quantities at the gastric level. Pizzas, like all solid matrices, release phenolic compounds with difficulty. It is necessary to extract them, to increase their bioaccessibility and potentially bioavailability. The acidic $\mathrm{pH}$ at the gastric level and the alkaline $\mathrm{pH}$ of the intestine influence the extraction of phenolic compounds. The bioaccessibility of the phenolic fraction of pizza "marinara TSG" was higher than other similar pizzas [38]. Moreover, the potential of pizza nutraceuticals is linked to the tomato quality and the lycopene they contain $[39,40]$. Bioaccessibility improves when tomatoes are turned into sauce, as the cells break down, and the release of lycopene is facilitated. Other factors that facilitate release are the heat treatments that can destroy the cell walls membrane [41]. and denature carotene-protein interactions $[42,43]$. In the case of pizzas, the rapid heat treatment does not allow the complete breakage of the membrane, but the formation of micelles with the oil lipids facilitates the lycopene release. In this work, the antioxidant activity of the carotenoid extract increased when pizza was prepared with oil addition, and it was higher in the pizza "marinara TSG." The enhance of antioxidant activities in processed foods could be linked to the botanicals liberated from the matrix during processing [44], the formation of the Maillard products [45] deactivation of the endogenous oxidative enzymes, and polymerization due to heating [46]. This result was probably due to the mutual protection of tomato and olive oil in the "Pizza Napoletana", both rich in antioxidant compounds [47]. The oil on the pizzas products dissolves the lycopene contained in tomato, which is insoluble in the crystalline form [48]. Moreover, during the cooking process, the flavonoid glycosides of the EVOO, form free hydroxyl phenol [49-51], able to protect lycopene from thermal oxidation. The bioaccessibility level of lycopene was higher in pizza "marinara TSG" than other similar products. The bile acids and the pancreatin contribute to the absorption of the lycopene, incorporating it into micelles and making it available for absorption [52]. The quality of the oil used to prepare pizza had an impact on the lycopene bioaccessibility. The EVOO resulted in the highest lycopene bioaccessibility. Lipids rich in C12:0 fatty acids determine lower bioaccessibility of the lycopene than lipids containing many 18:1 fatty acid (EVOO and olive oil). The first fatty acids form with monoglycerides, weakly swollen micelles, which make lycopene not very soluble [53]. Moreover, the EVOO offers the necessary environment for the isomerization of the lycopene. Short cooking time and the use of the EVOO enhance the lycopene Z-isomers formation [54]. The absorption, the transport flexibility, and the antioxidant capacity of the Z-isomers are higher than Eisomers [55]. This hypothesis follows a previous study, which showed that the co-digestion of tomato sauce with different added oils caused the higher lycopene bioaccessibility when EVOO was added [56]. Moreover, Tulipani et al.[57], hypothesized that the lipid matrix in the sauces might stimulate the re-absorption events by enterohepatic circulation, potentially affecting the apparent plasma halflife of these compounds.

\section{Conclusions}

This study investigated the nutraceutical potential of pizza marinara listed in the register of specialties guaranteed (TSG) of the E.U. In particular, the concentration of polyphenols and lycopene (known antioxidant molecule) in the pizza " marinara TSG" and their bioavailability in the human body were assessed for the first time. The results were compared with those obtained by analyzing the same parameters in the pizzas "marinara" prepared in a way that does not comply with the TSG specification and without oil added. Our results showed unequivocally that the pizza "marinara TSG" had the highest polyphenols and lycopene content compared to other "marinara" pizzas and that the mix of ingredients used for its preparation contributed to making the lycopene particularly bioavailable for our health. Moreover, the pizza "marinara TSG" showed the highest levels of antioxidant activity and the highest bioaccessibility of the phenols and lycopene. 
The 1st International Electronic Conference on Antioxidants in Health and Disease, 1-15 December 2020

In conclusion, our results confirm the nutritional importance of traditional preparations and demonstrate the functional potential of the pizza "marinara TSG" as a food rich in bioavailable antioxidants. Our data invite a higher consumption of traditional "pizza Napoletana", a food rich in readily bioaccessible antioxidant compounds, and confirm that improper use of the name "pizza" could damage the reputation of the authentic product and determine economic damage. This data could be used to write a nutraceutical label on traditional pizza to help consumers to make informed pizza selections. Historic craft, the know-how of pizza baking, rigorous rules, best-quality of local raw products are reliable drivers of success of the traditional pizza "marinara TSG".

Author Contributions: formal analysis, L.I.; data curation, G.G.; formal analysis, writing-review, and editing, I.D.; project administration A.R. All authors have read and agreed to the published version of the manuscript.

Conflicts of Interest: The authors declare no conflict of interest.

\section{References}

1. Nowak, Z.B. Folklore, fakelore, history: Invented tradition and the origins of the pizza margherita. Food Culture Soc. 2014, 17, 103-124.

2. European Commission. Regulation No 97/2010 of February 42010 entering name in the register of traditional specialties guaranteed [Pizza Napoletana(S.T.G.)]. Off. J. Eur.Union 2010, L34, 7-16.

3. MiPAAF Ministero Politiche Agricole Alimentari e Forestali 16th Revision of list "Prodotti Agroalimentari Tradizionali", Gazzetta Ufficiale n.143 del 21 giugno 2016. 2016. Available online: https://www.politicheagricole.it/flex/cm/pages/ServeBLOB.php/L/IT/IDPagina/10241 (accessed on 2 May 2018).

4. Trichopoulou, A.; Vasilopoulou, E.; Georga, K.; Soukara, S.; Dilis, V. Traditional foods: Why and how to sustain them. Trends Food Sci. Technol. 2006, 17, 498-504.

5. Trichopoulou, A.; Soukara, S.; Vasilopoulou, E. Traditional foods: A science and society perspective. Trends Food Sci. Technol. 2007, 18, 420-427.

6. Fabiani, R.; Rosignoli, P.; de Bartolomeo, A.; Fuccelli, R.; Servili, M.; Montedoro, G.F.; Morozzi, G.; Oxidative, D.N.A. Damage is prevented by extracts of olive oil, hydroxytyrosol, and other olive phenolic compounds in human blood mononuclear cells and HL60 cells. J. Nutr. 2008, 138, 1411-1416.

7. Tundis, R.; Loizzo, M.; Menichini, F.; Statti, G.; Menichini, F. Biological and pharmacological activities of iridoids: Recent developments. Mini Rev. Med. Chem. 2008, 8, 399-420.

8. Dini, I.; Laneri, S. Nutricosmetics: A brief overview. Phytother. Res. 2019, 33, 3054-3063.

9. Goodwin, T.W. The carotenoids of the flower petals of Calendula officinalis. Biochem. J. 1954, 58, 90-94.

10. Drewnowski, A. Sensory properties of fats and fat replacements. Nutr. Rev. 2009, 50, 17-20.

11. Sandrou, D.K.; Arvanitoyannis, I.S. Low-fat/calorie foods: Current stateand perspectives. Crit. Rev. Food Sci. Nutr. 2000, 40, 427-447.

12. Crespo, M.C.; Tomé-Carneiro, J.; Dávalos, A.; Visioli, F. Pharma-Nutritional Properties of Olive Oil Phenols. Transfer of New Findings to Human Nutrition. Foods 2018, 7, 90.

13. Zarrouk, A.; Martine, L.; Grégoire, S.; Nury, T.; Meddeb, W.; Camus, E.; Badreddine, A.; Durand, P.; Namsi, A.; Yammine, A.; et al. Profile of fatty acids, tocopherols, phytosterols and polyphenols in mediterranean oils (argan oils, olive oils, milk thistle seed oils and nigella seed oil) and evaluation of their antioxidant and cytoprotective activities. Curr. Pharm. Des. 2019, 25, 1791-1805.

14. Obied, H.K. Biography of biophenols: Past, present and future. Funct. Foods Health Dis. 2013, 3, 230, doi:10.31989/ffhd.v3i6.51.

15. De Alzaa, F.; Guillaume, C.; Ravetti, L. Evaluation of chemical and physical changes in different commercial oils during heating. Acta Sci. Nutr. Health 2018, 2, 2-11.

16. Zhang, Q.; Saleh, S.M.; Cheng, J.; Shen, Q. Chemical alterations taken place during deep-fat frying based on certain reaction products: A review. Chem. Phys. Lipids 2012, 165, 662-681.

17. Dini, I.; Graziani, G.; Fedele, F.L.; Sicari, A.; Vinale, F.; Castaldo, L.; Ritieni, A. Effects of Trichoderma biostimulation on the phenolic profile of extra-virgin olive oil and olive oil by-products. Antioxidants 2020, 9, 284, doi:10.3390/antiox9040284. 
The 1st International Electronic Conference on Antioxidants in Health and Disease, 1-15 December 2020

18. Dini, I.; Seccia, S.; Senatore, A.; Coppola, D.; Morelli, E. Development and Validation of an Analytical Method for Total Polyphenols Quantification in Extra Virgin Olive Oils. Food Anal. Methods 2020, 13, 457464.

19. Soriguer, F.; Rojo-Martínez, G.; Dobarganes, M.C.; García Almeida, J.M.; Esteva, I.; Beltrán, M.; Ruiz De Adana, M.S.; Tinahones, F.; Gómez-Zumaquero, J.M.; García-Fuentes, E.; et al. Hypertension is related to the degradation of dietary frying oils. Am. J. Clin. Nutr. 2003, 78, 1092-1097.

20. Rodrigo, R.; Prat, H.; Passalacqua, W.; Araya, J.; Guichard, C.; Bachler, J.P. Relationship be-tween oxidative stress and essential hypertension. Hypertens. Res. 2007, 30, 1159-1167.

21. Ono, Y.; Mizuno, K.; Takahashi, M.; Miura, Y.; Watanabe, T. Suppression of advanced glycation and lipoxidation end products by Angiotensin II type-1 receptor blocker candesartan in type 2 diabetic patients with essential hypertension. Fukushima J. Med. Sci. 2013, 59, 69-75.

22. Baradaran, A.; Nasri, H.; Rafieian-Kopaei, M. Oxidative stress and hypertension: Possibility of hypertension therapy with antioxidants. J. Res. Med. Sci. 2014, 19, 358-367.

23. Adam, S.K.; Das, S.; Soelaiman, I.N.; Umar, N.A.; Jaarin, K. Consumption of repeatedly heated soy oil increases serum parameters related to atherosclerosis in ovariectomized rats. Tohoku J. Exp. Med. 2008, 215, 219-226.

24. Adam, S.K.; Das, S.; Jaarin, K.A. detailed microscopic study of the changes in the aorta of experimental model of postmenopausal rats and with repeatedly heated palm oil. Int. J. Exp. Pathol. 2009, 90, 321-327.

25. Ng, C.Y.; Leong, X.F.; Masbah, N.; Adam, S.K.; Yusof, K.; Jaarin, K. Heated vegetable oils and cardiovascular risk factors. Vascul. Pharmacol. 2014, 61, 1-9.

26. Mathias, K.S.; Russel, G.F. Effects of processing on tomato bioactive volatile compounds. Bioact. Compd. Foods 2002, 12, 155-172.

27. Gahler, S.; Konrad, O.; Bohm, V. Alterations of vitamin C, total phenolics, and antioxidant capacity as affected by processing tomatoes to different products. J. Agric. Food Chem. 2003, 51, 7962-7968.

28. Manzo, N.; Santini, A.; Pizzolongo, F.; Aiello, A.; Romano, R. Degradation kinetic (D100) of lycopene during the thermal treatment of concentrated tomato paste. Nat. Prod. Res. 2018, 21, 1-7.

29. Grabowska, M.; Wawrzyniak, D.; Rolle, K.; Chomczynski, P.; Oziewicz, S.; Jurgaand, S.; Barciszewski, J. Let food be your medicine: Nutraceutical properties of lycopene. Food Funct. 2019, 10, 3090-3102.

30. Gao, X.; Bjork, L.; Trajkovski, V.; Uggla, M. Evaluation of antioxidant activities of rosehip ethanol extracts in different test systems. J. Agric. Food Chem. 2000, 80, 2021-2027.

31. Re, R.; Pellegrini, N.; Proteggente, A.; Pannala, A.; Yang, M.; Rice Evans, C. Antioxidant activity applying an improved ABTS radical cation decolorization assay. Free Rad. Biol. Med. 1999, 26, 1231-1237.

32. Raiola, A.; Meca, G.; Mañes, J.; Ritieni, A. Bioaccessibility of Deoxynivalenol and its natural co-occurrence with Ochratoxin A and Aflatoxin B 1 in Italian commercial pasta. Food Chem. Toxic. 2012, 50, $280-287$.

33. Obied, H.K. Biography of biophenols: Past, present and future. Func. Foods Health Dis. 2013, 3, $230-241$.

34. Dini, I.; Graziani, G.; Gaspari, A.; Fedele, F.L.; Sicari, A.; Vinale, F.; Cavallo, P.; Lorito, M.; Ritieni, A. New Strategies in the Cultivation of Olive Trees and Repercussions on the Nutritional Value of the Extra Virgin Olive Oil. Molecules 2020, 25, 2345.

35. Cavallo, P.; Dini, I.; Sepe, I.; Galasso, G.; Fedele, F.L.; Sicari, A.; Bolletti Censi, S.; Gaspari, A.; Ritieni, A.; Lorito, M.; et al. An Innovative Olive Pâté with Nutraceutical Properties. Antioxidants 2020, 9, 581.

36. Caporaso, N.; Panariello, V.; Sacchi, R. The " true" Neapolitan pizza: Assessing the influence of extra virgin olive oil on pizza volatile compounds and lipid oxidation. J. Culin. Sci. Technol. 2015, 13, $29-48$.

37. Miro-Casas, E.; Covas, M.; Farre, M.; Fito, M.; Ortuño, J.; Weinbrenner, T.; Roset, P.; de la Torre, R. Hydroxytyrosol disposition in humans. Clin. Chem. 2003, 49, 945-952.

38. Rubió, L.; Serra, A.; Oliver Chen, C.-Y.; Macià, A.; Covas, M.I.; Sola, R.; Motilva, M.J. Effect of the cooccurring components from olive oil and thyme extracts on the antioxidant status and its bioavailability in an acute ingestion in rats. Food Funct. 2014, 5, 740-747.

39. Müller, L.; Caris-veyrat, C.; Lowe, G.; Böhm, V. Lycopene and its antioxidant role in the prevention of cardiovascular diseases-A critical review. Crit. Rev. Food Sci. Nutr. 2016, 56, 1868-1879.

40. El-raey, M.A.; Ibrahim, G.E.; Eldahshan, O.A. lycopene and lutein; a review for their chemistry and medicinal uses. J. Pharmacogn. Phytochem. 2013, 2, 245-254. 
The 1st International Electronic Conference on Antioxidants in Health and Disease, 1-15 December 2020

41. Holzenburg, J.A.; King, S. Physical barriers to carotenoid bioaccessibility Ultrastructure survey of chromoplast and cell wall morphology in nine carotenoid-containing fruits and vegetables. J. Sci. Food Agric. 2012, 92, 2594-2602.

42. Rich, G.T.; Bailey, A.L.; Faulks, R.M.; Parker, M.L.; Wickham, M.S.J.; Fillery-Travis, A. Solubilization of carotenoids from carrot juice and spinach in lipid phases: I. Modeling the gastric lumen. Lipids 2003, 38, 933-945.

43. Thane, C.; Reddy, S. Processing of fruit and vegetables: Effect on carotenoids. Food Sci. Nutr. 1997, 97, 5865.

44. Dewanto, V.; Wu, X.; Adom, K.K.; Liu, R.H. Thermal processing enhances the nutritional value of tomatoes by increasing total antioxidant activity. J. Agric. Food Chem. 2002, 50, 3010-3014.

45. Nicoli, M.C.; Anese, M.; Parpinel, M. Influence of processing on the antioxidant properties of fruit and vegetables. Trends Food Sci. Technol. 1999, 10, 94-100.

46. Pinelo, M.; Manzocco, L.; Nuñez, M.J.; Nicoli, M.C. Interaction among phenols in food fortification: Negative synergism on antioxidant capacity. J. Agric. Food Chem. 2004, 52, 1177-1180.

47. Yu, J.; Gleize, B.; Zhang, L.; Caris-Veyrat, C.; Renard, C.M.G.C. Heating tomato puree in the presence of lipids and onion: The impact of onion on lycopene isomerization. Food Chem. 2019, 296, 9-16.

48. De Alvarenga, J.F.R.; Tran, C.; Hurtado-Barroso, S.; Martinez-Huélamo, M.; Illan, M.; Lamuela-Raventos, R.M. Home cooking and ingredient synergism improve lycopene isomer production in Sofrito. Food Res. Int. 2017, 99, 851-861.

49. Orsavova, J.; Misurcova, L.; Ambrozova, J.; Vicha, R.; Mlcek, J. Fatty acids composition of vegetable oils and its contribution to dietary energy intake and dependence of cardiovascular mortality on dietary intake of fatty acids. Int. J. Mol. Sci. 2015, 16, 12871-12890.

50. Medina, E.; de Castro, A.; Romero, C.; Brenes, M. Comparison of the Concentrations of Phenolic Compounds in Olive Oils and Other Plant Oils: Correlation with Antimicrobial Activity. J. Agric. Food Chem. 2006, 54, 4954-4961.

51. Sandberg, A.S. Methods and options in vitro dialyzability; benefits and limitations. Int. J. Vitam Nutr. Res. 2005, 75, 395-404.

52. Colle, I.J.P.; Lemmens, L.; Van Buggenhout, S.; Met, K.; Van Loey, A.M.; Hendrickx, M.E. Processing tomato pulp in the presence of lipids: The impact on lycopene bioaccessibility. Food Res. Inter. 2013, 51, 32-38.

53. Porter, C.J.H.; Kaukonen, A.M.; Taillardat-Bertschinger, A.; Boyd, B.J.; O'Connor, J.M.; Edwards, G.A.; Edwards, G.A.; Charman, W.N. Use of in vitro lipid digestion data to explain the in vivo performance of triglyceride-based oral lipid formulations of poorly water-soluble drugs: Studies with halofantrine. J. Pharm. Sci. 2004, 93, 1110-1121.

54. Honda, M.; Horiuchi, I.; Hiramatsu, H.; Inoue, Y.; Kitamura, C.; Fukaya, T.; Takehara, M. Vegetable oilmediated thermal isomerization of (all-E)-lycopene: Facile and efficient production of Z-isomers. Eur. J. Lipid Sci. Technol. 2016, doi:10.1002/ejlt.201500446.

55. Colle, I.J.P.; Lemmens, L.; Tolesa, G.N.; Van Buggenhout, S.; De Vleeschouwer, K.; Van Loey, A.M.; Hendrickx, M.E. Lycopene Degradation and Isomerization Kinetics during Thermal Processing of an Olive Oil/Tomato Emulsion. J. Agric. Food Chem. 2010, 58, 12784-12789.

56. Colle, I.J.P.; Van Buggenhout, S.; Lemmens, L.; Van Loey, A.M.; Hendrickx, M.E. The type and quantity of lipids present during digestion influence the in vitro bioaccessibility of lycopene from raw tomato pulp. Food Res. Int. 2012, 45, 250-255.

57. Tulipani, S.; Martinez Huelamo, M.; Rotches Ribalta, M.; Estruch, R.; Ferrer, E.E.; Andres-Lacueva, C.; Illan, M.; Lamuela-Raventós, R.M.L. Oil matrix effects on plasma exposure and urinary excretion of phenolic compounds from tomato sauces: Evidence from a human pilot study. Food Chem. 2012, 130, 581-590.

Publisher's Note: MDPI stays neutral with regard to jurisdictional claims in published maps and institutional affiliations.

(C) 2020 by the authors. Licensee MDPI, Basel, Switzerland. This article is an open access article distributed under the terms and conditions of the Creative Commons Attribution (CC BY) license (http://creativecommons.org/licenses/by/4.0/). 